\title{
On the applicability of dye penetrant tests on vacuum components - allowed or forbidden?
}

\author{
Michael Schröder, Christoph Biedermann, Reinhard Vilbrandt
}

\author{
Max-Planck-Institut für Plasmaphysik, EURATOM Association, Teilinstitut Greifswald, Wendelsteinstr. 1, 17491 \\ Greifswald, Germany\#
}

The penetrant testing (PT) is a common non-destructive procedure for the testing of components and in particular of welds. With PT it is possible to detect surface imperfections (e.g. cracks) which have a special potential to lead to the failure of the component or of the weld. PT is substantially more sensitive than a purely visual examination.

Because the complicated geometries of fusion experiments make the accessibility for repairs during the operation extremely difficult, very high efforts on testing with sensitive procedures, for instance with dye penetrant testing during assembly is required. In contrast to this desire for widespread penetrant testing, however, is the general fear that dye penetrant tested components or welds, which are used in the vacuum, are contaminated by the dye in such a way that they do not fulfill the cleanliness requirements for vacuum components. Therefore dye penetrant testing of such vacuum components is usually considered problematic.

This study aims to clarify the applicability of dye penetrant tests on components exposed to high- vacuum. Recommendations are formulated concerning the PT procedure of vacuum components and the cleaning procedures for penetrant tested areas under vacuum necessary after a dye penetrant test.

Keywords: dye penetrant testing, vacuum component, UHV, NDT

\section{Background}

Fusion experiments are complex equipments with often complicated geometry. It is desirable to operate the machines over long time periods without shut-down interruptions for repair or service. If there are interruptions of the regular experiment operation, the search for failures and the repair will be very difficult and extensive in time and costs. To avoid these kinds of breakdowns, a strong effort on quality control is necessary like e. g. non-destructive testing procedures during the manufacturing of components and its assembly. Beside the visual examination (VT), the method of penetrant testing (PT) is often most common non-destructive procedure for the testing of surfaces of components and in particular of welds.

In contrast to this desire for widespread penetrant testing, however, there is the general fear that components or welds tested with the dye penetrant and which are used in the environment of cryo-vacuum and particularly in the ultra-high vacuum conditions are contaminated by the dye in such a way that they do not fulfill the cleanliness requirements for vacuum components. This fear is mainly based on the high penetrating property of the dye and its particular creeping characteristics, which just is the property required for the test procedure. After the penetration test, however, the penetrant dye may remain in cavities like porosities or in cracks and might cause virtual leaks during the evacuation and in the vacuum, due to extended outgassing. As the penetrant dye primarily consists of hydrocarbons, which are considered critical for outgassing in fusion devices, a penetrant test of such vacuum components is usually considered problematic.
In this investigation we try to quantify the related properties of a penetrant dye used during the assembly of the advanced stellarator Wendelstein 7-X [1]. The possible blocking of leaks by the PT medium was not investigated, because at the W7-X all PT tested vacuum boundary welds are leak tested before the PT application.

\section{Approach and test procedure}

Particular samples with defined surface finishes were PT tested, cleaned and then the residual gas of the probes was analyzed with a mass spectrometer in a vacuum chamber. Thereby the influence of the material and the surface treatment was tested and how the penetrant might change the composition of the residual gas under vacuum. The following aspects can be relevant:

- $\quad$ kind of test surface

- geometry of tested object

- $\quad$ existing imperfections on the surface

- cleaning procedures used after PT

- $\quad$ heating and bake-out of the test object after PT

- composition of the test medium

\subsection{Test samples}

Test samples (dimension 150x55x10 mm) were made of austenitic steel 1.4429 with different surfaces defined by manufacturing. The surface of one side of the block (150x55 mm, test surface) was modified. All the other surfaces were milled. The following test surfaces with increasing structuring of the surface were tested (fig. 1): 
- $\quad$ sample No. 1: milled surface (roughness Ra = $0,15 \mu \mathrm{m})$

- $\quad$ sample No. 3: as-rolled plate $(\mathrm{Ra}=4,81 \mu \mathrm{m})$

- $\quad$ sample No. 5: smooth tungsten-inert gas (TIG) build-up welding

- $\quad$ sample No. 6: rough TIG build-up welding

- $\quad$ sample No. 7: smooth metal active-gas (MAG) build-up welding

- $\quad$ sample No. 8: rough MAG build-up welding

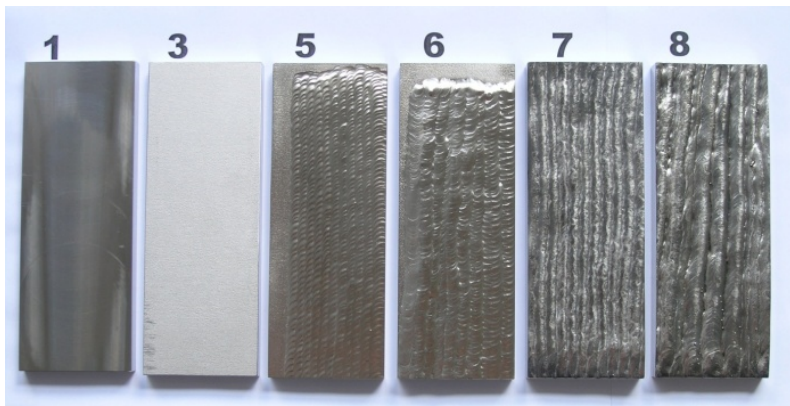

Fig.1 photograph of the different surfaces used as samples in this investigation

Before the tests were performed, every sample was cleaned in an ultrasonic bath, washed up with distilled water, dried with hot air and wiped with ethanol. This is the regular procedure applied for metal cleaning for ultra-high vacuum application.

\subsection{Application of the PT and test results}

The samples were PT tested according to DIN EN 571-1 [2] (test medium system IICd). The time for penetration and processing was in each case $30 \mathrm{~min}$. The test results are listed in Table 1.

Tab. 1 Results of the penetration tests

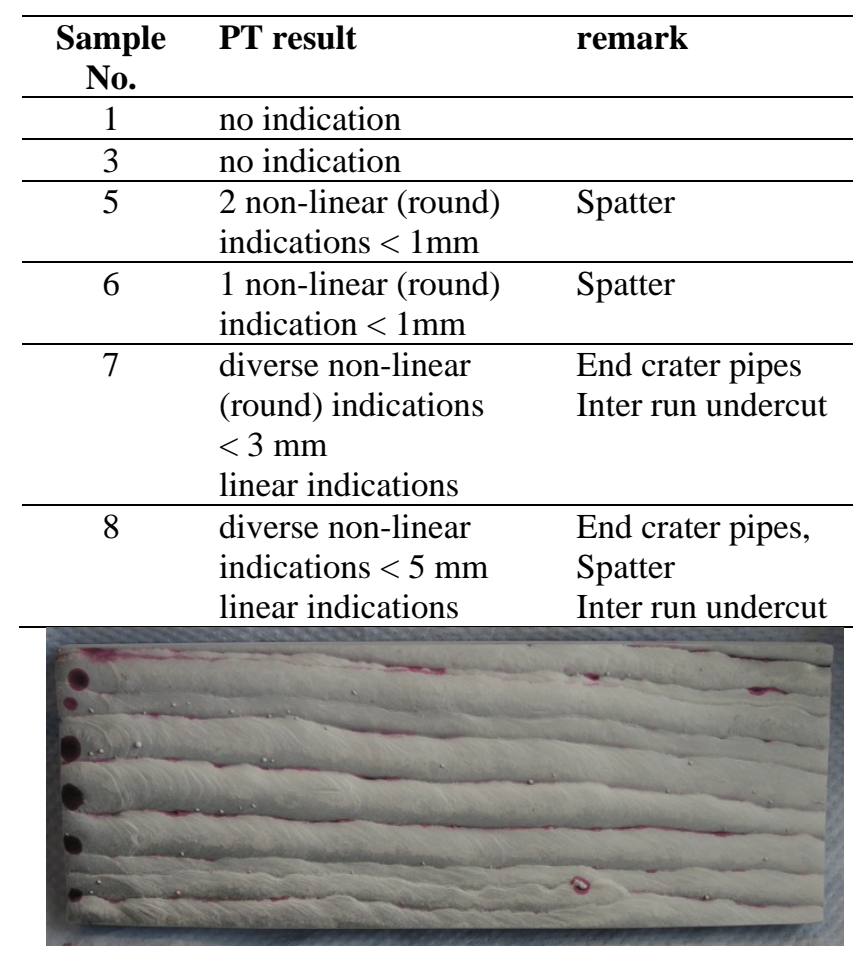

Fig. 2: Sample No. 8 after PT

\subsection{Testing medium}

The Helling's Nordtest series was used as PT testing medium. This series consists of the penetrant U88, cleaner U87 and developer U89. The detailed composition of the medium was not available from the company. Only the information of the material safety data sheets [3] is available. According to this the penetrant consists of fatty acid ester (hydrocarbon) and the diazo dye Solvent Red 164 (a compound of hydrocarbon and nitrogen). The cleaner medium consists of ethanol and the developer consists of a suspension of inorganic solids in ethanol. Cleaner and developer are in an aerosol (injecting fluid: propane, butane). Only the penetrant is critical for the contamination of component parts because of its high penetrating property.

Further testing media were not tested.

\subsection{Cleaning processes after PT}

After the PT execution the samples were cleaned with the following procedures respectively:

A) cleaner U87 and cloth, cryo blasting with CO2, drying with hot air, ethanol

B) cleaner U87 and cloth, drying in the air

C) In between cleaning after the penetration process with cleaner U87 and cloth (in this case without the later application of the developer) -thereby typically remains of the penetrant stay on the surface, especially at rough surfaces

It is expected that the cleaning effect is most efficient with procedure A) and is the worst with $\mathrm{C}$ ).

\subsection{Test rig}

A schematic of the vacuum chamber and equipment is shown in fig. 3 .

The test chamber could be baked with heating bands which were placed on the outside of the vacuum chamber. The temperature of $150^{\circ} \mathrm{C}$ is the standard bakeout temperature to be applied for the UHV components at W7-X. The heat was mostly transferred to the sample by thermal radiation. So the samples were not as hot as the chamber wall set to $150^{\circ} \mathrm{C}$. 


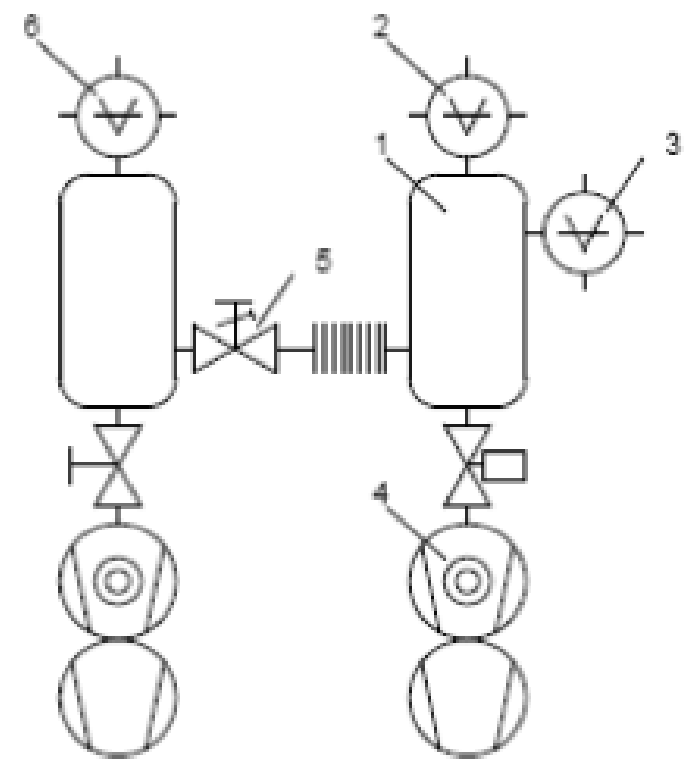

Fig. 3: Test rig in principle

1 Test chamber (total volume approximately $3 \mathrm{l}$ )

2 Pirani vacuum gauge

3 Hot cathode vacuum gauge

4 Turbomolecular pump (pumping speed 64l/s)

5 Hand valve

6 Quadrupole Mass Analyzer QMS200 in a separate vacuum chamber with a turbo pump (pumping speed 2301/s)

\section{Results and interpretation}

\subsection{Mass spectra at room temperature}

To show the basically negative influence of the penetrant to the vacuum environment, the sample No. 8 was investigated in the mass spectrometer after a particular insufficient cleaning procedure whereby rests of the penetrant were clearly visible. The typical fragments of hydrocarbons at mass numbers between 45 and 100 are clearly visible, see fig. 4 .

\subsubsection{Comparison of different surfaces}

A comparison of the outgassing of the different tested surfaces after the PT application and cleaning in the same way showed no significant differences. The surfaces of all samples could be sufficiently cleaned. Of course the cleaning of rough surfaces was more extensive than of smooth ones.

\subsubsection{Comparison before and after bake-out}

Fig. 5 shows the sample no. 5, which was cleaned with the procedure $B$ (ср. 2.4). The mass spectra before and after a bake-out sequence $\left(3.5 \mathrm{~h}\right.$ at $\left.150^{\circ} \mathrm{C}\right)$ are shown. Clearly the improved residual spectrum and cleaning effect is visible.

\subsubsection{Comparison of different cleaning procedures}

A comparison of the outgassing of samples with applied different cleaning procedures after the PT mentioned in 2.4. has shown no essential influence of the cleaning procedures. All surfaces could sufficiently be cleaned with cleaner and cloth. The cryo blasting can improve the results, but the surfaces may become wet due to condensation of humidity after extensive surface cooling by the cryo blasting. The samples have to be dried afterwards.

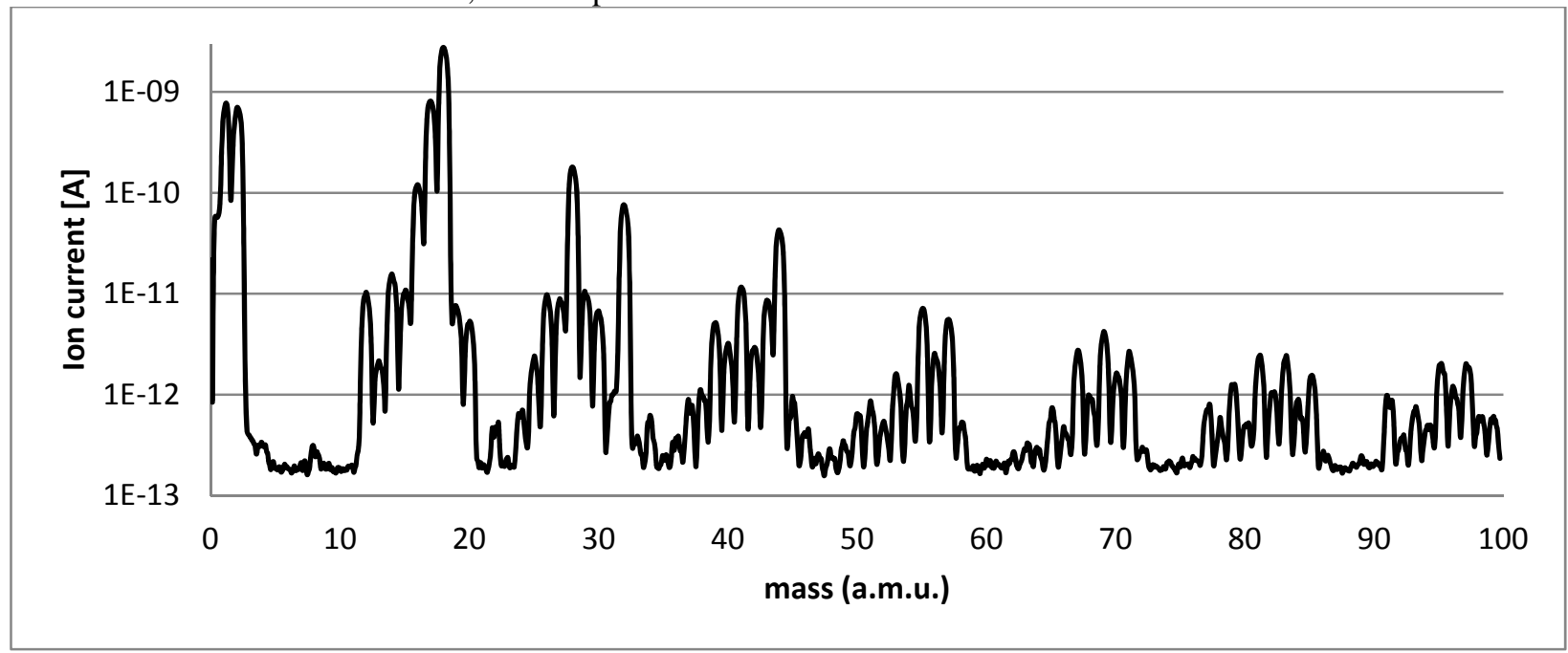

Fig. 4: Mass spectrum sample 8 with contamination by penetrant

\subsection{Mass spectra at higher temperatures}

After the PT well cleaned samples, which don't outgas at room temperature, show a different behavior at increased temperature. Already at $40^{\circ} \mathrm{C}$ these samples start to outgas strongly. This effect continues with a further increase of the temperature. Nevertheless, after3 to 4 hours of bake-out at $150^{\circ} \mathrm{C}$ the pollutions have disappeared, even at samples with normally unacceptable PT indications like at the samples no. 7 and 
no. 8. Even so the temperature of the samples did not reach $150^{\circ} \mathrm{C}$ applied to the chamber wall, the cleaning process was sufficient. Fig. 6 shows the mass spectra of the sample no. 7 at a temperature of the vacuum chamber of $150^{\circ} \mathrm{C}$ immediately after the achievement of this temperature and after 4 hours baking-out at $150^{\circ} \mathrm{C}$.

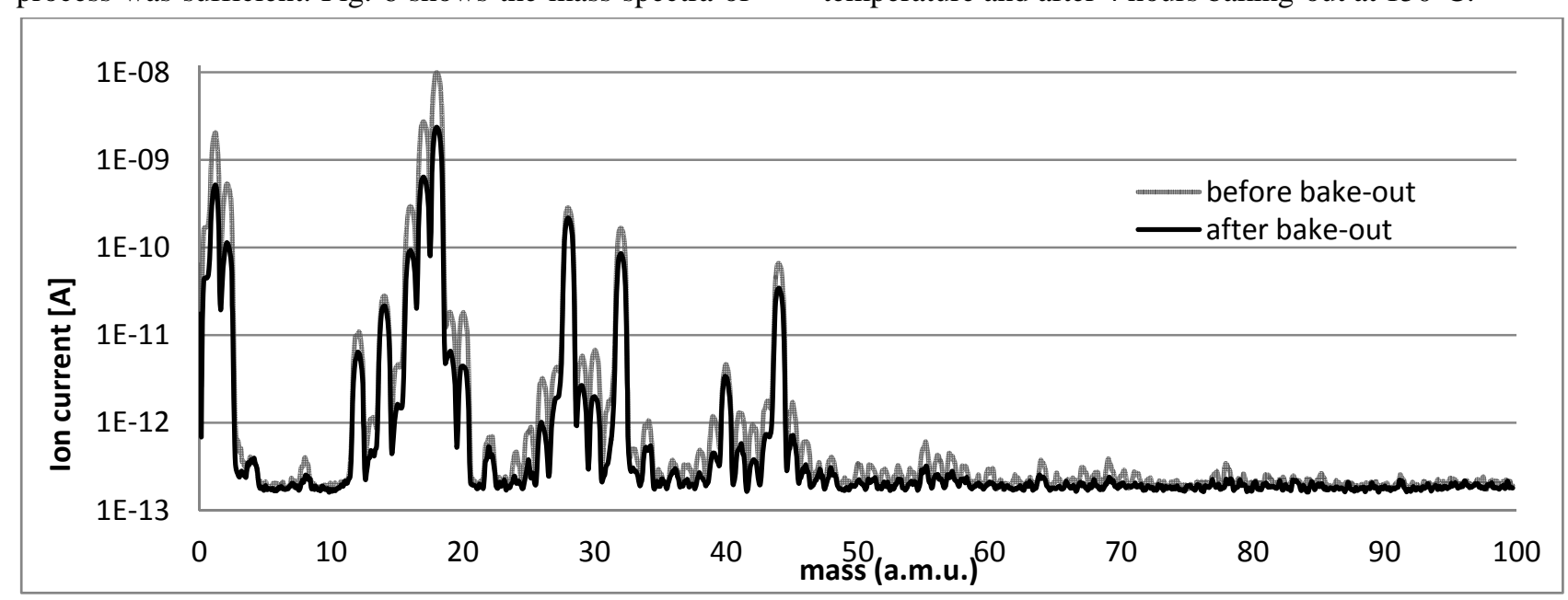

Fig. 5: Mass spectra of sample 5 before and after bake-out

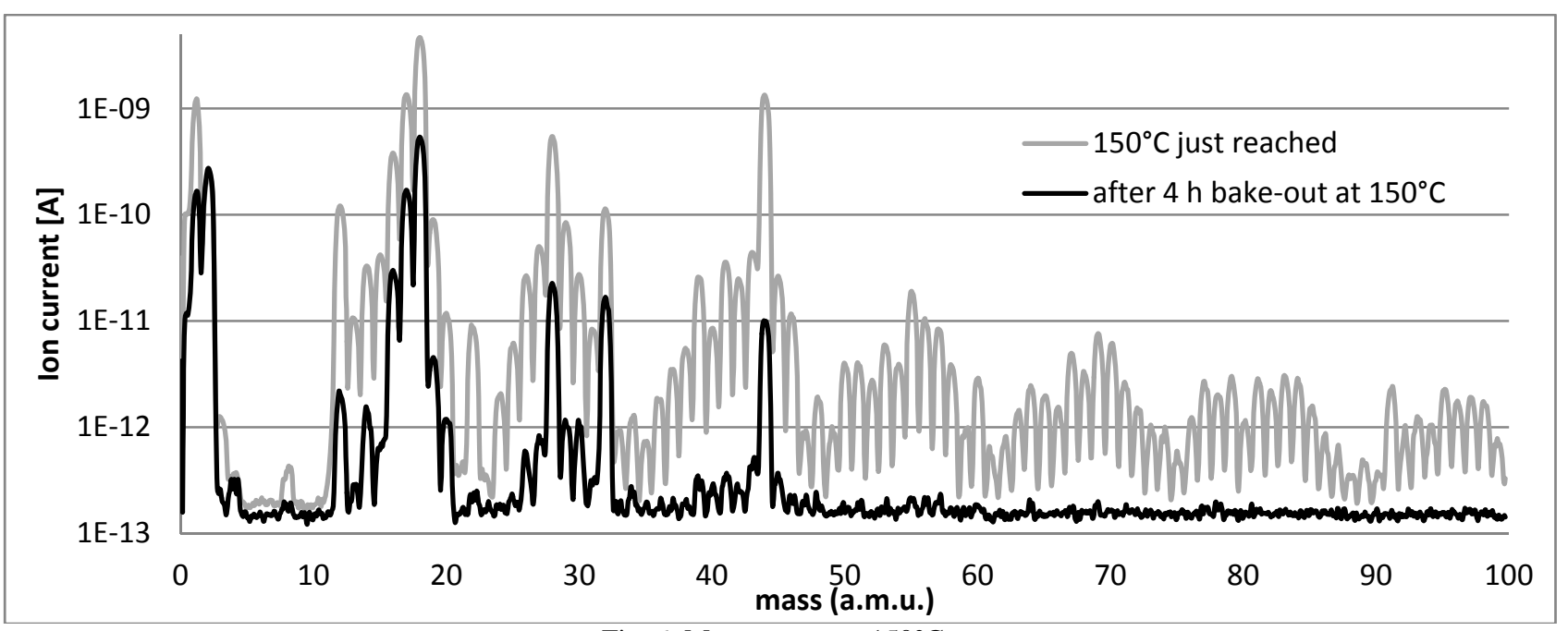

Fig. 6: Mass spectra at $150^{\circ} \mathrm{C}$

\section{Conclusions for the PT application}

The results of the investigations show, that the PT application on components for use under vacuum conditions can in general be allowed. The following details should be adhered to:

The test surface should have a simple geometry. In no case they should have gaps or holes, in which the penetrant can get.

Vacuum boundary welds have to be leak tested before PT application. So there is no risk of subsequent leaks which are blocked by the PT medium before.

With PT detached small indications, that are acceptable in accordance to established rules (e. g. DIN EN 23277, allowable limit 1 or AD2000 HP 5/3) could be left. Larger indications, which could include a sizeable portion of penetrant, should be grinded and repaired.
An efficient cleaning after PT is necessary. But obviously an efficient cleaning with ethanol and cloth until no contaminations are visible is sufficient.

In comparison to MAG weld seams, TIG weld seams are basically more convenient to PT application and the cleaning is easier afterwards. If PT is foreseen TIG should used as the welding procedure.

PT tested components should be baked out after the cleaning in a vacuum chamber at min. $150^{\circ} \mathrm{C}$, especially if the components should be used at high temperatures later. For simple components few hours are sufficient.

This investigation was performed with one special PT medium. Other test media may show a different behavior and therefore, they should be investigated in a similar way before they are used for vacuum- or UHVcomponents.

\section{References}

[1] H.-S. Bosch, V. Erckmann, R. König, F. Schauer, R. Stadler, A. Werner, Wendelstein 7-X Team; 
Construction of Wendelstein 7-X - engineering a steadystate stellarator. , IEEE Trans. Plasma Science, 38.

[2] DIN EN 571-1 : Non-destructive testing, Penetrant testing,

[3] Sicherheitsdatenblatt - NORD-TEST Kontrastrot U88 
